\title{
FALLAMIENTO Y SISMICIDAD DEL ÁREA ENTRE CARTAGO Y SAN JOSÉ, VALLE CENTRAL DE COSTA RICA
}

\author{
Mario Fernández ${ }^{1}$ \& Walter Montero ${ }^{2}$ \\ 1 Centro de Investigaciones Geofísicas (CIGEFI), Universidad de Costa Rica y Red \\ Sismológica Nacional (RSN: ICE-UCR) \\ 2 Escuela Centroamericana de Geología, Universidad de Costa Rica \\ y Red Sismológica Nacional (RSN: ICE-UCR)
}

(Recibido 17/12/99; Aceptado 12/4/02)

\begin{abstract}
Faulting and seismicity of an area located between Cartago and San José, in central Costa Rica, have been analyzed. Most of the area belongs to the Central Valley and only its southwestern part to the Talamanca Ridge. Faults were determined by neotectonic criteria, using mostly air photos and field reconnaissance. In order to look for the best correlation between faults and earthquakes, we selected the best events located between 1992 and 1996 by the Red Sismológica Nacional (ICE-UCR). The selected hypocenters show three main seismic clusters; one is located in the San Miguel-Higuito-Patarra (SMHP) area and correlates with the Agua Caliente fault. The second one is located near Escazú and can be related with the Belo Horizonte fault. The third one is located near Tobosi. High quality focal mechanisms from these seismic clusters, indicate different types of seismic slip.
\end{abstract}

RESUMEN: Se estudió la sismicidad y el fallamiento en un sector del centro de Costa Rica ubicado entre las ciudades de Cartago y San José. El área forma parte del Valle Central, aunque incluye una pequeña parte de las estribaciones de la Cordillera de Talamanca. Las fallas fueron evaluadas con criterios neotectónicos y para ello se utilizaron fotografías aéreas y reconocimiento de campo. Con el propósito de encontrar la mejor correlación posible entre fallas y temblores, se escogieron siguiendo diferentes criterios, los mejores eventos localizados entre 1992 y 1996 por la Red Sismológica Nacional (RSN, ICE-UCR). La sismicidad seleccionada muestra tres principales agrupamientos. Uno se ubica en el área San Miguel-Higuito-Patarrá (SMHP) y se asocia con la falla Agua Caliente. El segundo se ubica cerca de Escazú y se correlaciona con la falla Belo Horizonte. El tercero se localiza cerca de Tobosi. Mecanismos focales realizados para eventos de estas fuentes sísmicas indican diferentes tipos de deslizamientos en las fallas.

\section{INTRODUCCIÓN}

Históricamente el Valle Central de Costa Rica ha sido el escenario de destructivos temblores corticales de magnitudes intermedias $(5,0>$ Ms > 6,5) (Fig. 1). Entre los que han provocado efectos más dañinos destacan los que han ocurri- do cerca de la ciudad de Cartago y al sureste de San José. Estos temblores muestran un alto riesgo sísmico en el sureste del Valle Central, donde está el asiento de un importante sector de la población costarricense. De hecho, el crecimiento que han tenido las ciudades de San José y Cartago ha llevado a que áreas con fallamiento activo 


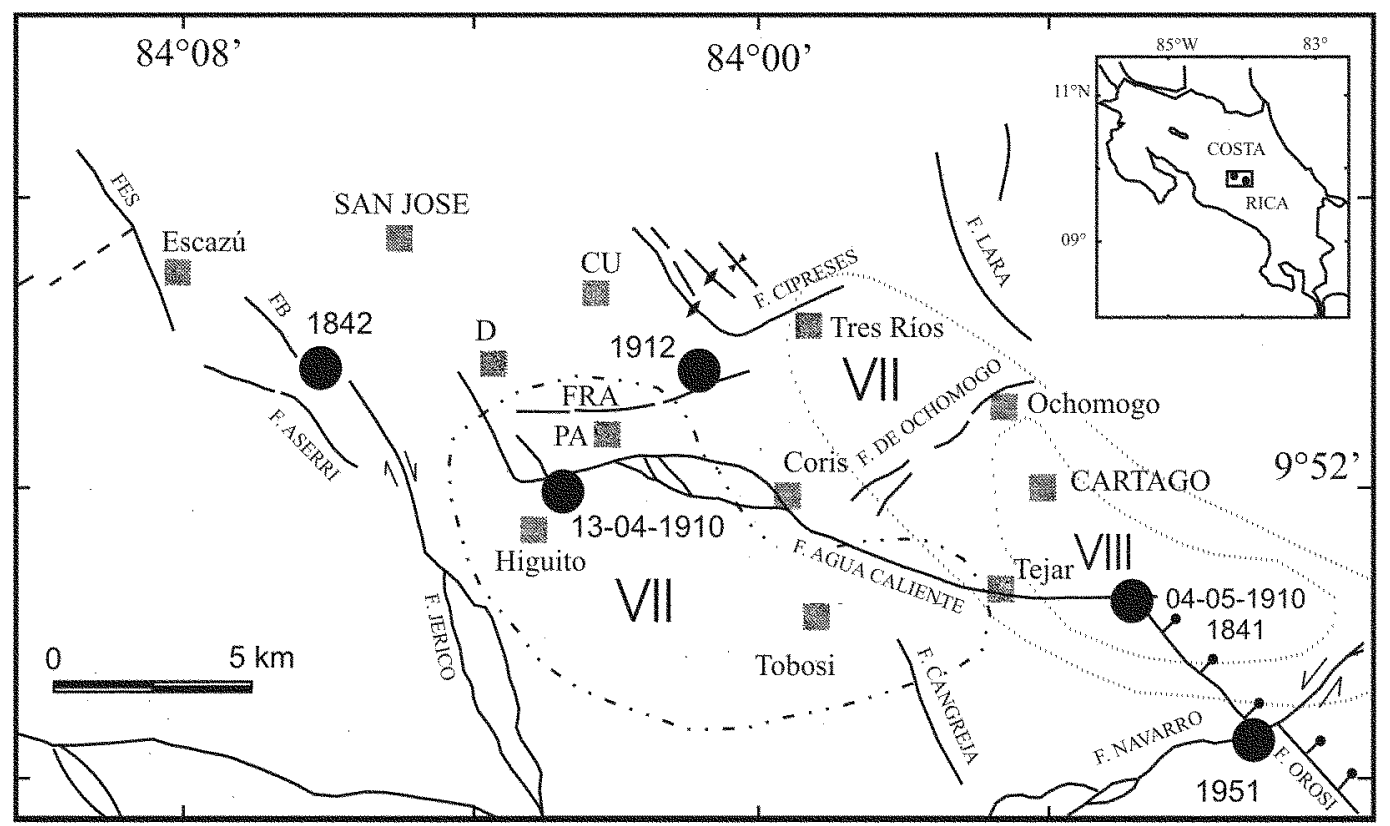

Fig. 1: Fallamiento neotectónico de la zona de estudio. Se indica tipo de movimiento para las fallas de desplazamiento de rumbo. Fallas con rayas cortas con círculo negro son fallas de desplazamiento normal e indican bloque que desciende. Anticlinales y sinclinales son las rayas con triángulos divergentes y convergentes respectivamente. Se muestran con círculos rellenos los epicentros de los terremotos destructivos ocurridos entre 1841 y 1951. Las fallas neotectónicas son FE: Falla Escazú, FB: Falla Belo Horizonte y FRA: Falla Río Azul. Las localidades son D: Desamparados y CU: Curridabat. Las áreas de intensidad $\geq$ VII se muestran para los terremotos de 1910.

estén siendo actualmente urbanizadas. Por esta razón consideramos, que es importante identificar y caracterizar el fallamiento del área, con el fin de que el fallamiento activo sea considerado en la planificación urbana futura. Por otro lado, el reconocimiento de las fuentes sísmicas es la base sobre la cual se apoyan los estudios de amenaza sísmica, los que proporcionan los parámetros de diseño para que las obras que se construyan en esta zona sean más seguras.

La sismicidad de esta zona fue estudiada previamente por Montero \& Dewey (1982) y por Montero \& Morales (1988). Estos últimos autores, además, hicieron una correlación preliminar entre los sismos históricos y el fallamiento neotectónico. Montero (1983) y Morales (1987) indican que los temblores de esta zona son de magnitud menor a 6,5 , profundidad menor a $20 \mathrm{~km}$ y han generado aceleraciones de las partículas del suelo entre 20 y $50 \%$ de la gravedad.
Debido al potencial sísmico de la zona y aprovechando que actualmente se cuenta con mejores localizaciones de temblores y un mejor conocimiento del fallamiento reciente, se ha considerado conveniente estudiar con detalle la sismicidad del área entre Cartago y San José y en particular determinar las fallas con las cuales se podría asociar la sismicidad histórica y reciente.

\section{METODOLOGÍA}

Inicialmente se recopiló la sismicidad del área para el período julio de 1976-octubre de 1981 de las siguientes publicaciones: Montero \& Dewey (1982), Montero \& Morales (1984) y Montero \& Alvarado (1995). Posteriormente se seleccionó la sismicidad superficial (profundidad menor a $30 \mathrm{~km}$ ) entre 1984-1996 de la base de temblores de la Red Sismológica Nacional (RSN: ICE-UCR). Considerando la necesidad de 
utilizar solamente las localizaciones de buena calidad, se escogieron solamente los temblores del período 1992 a 1996, con lecturas de onda P en las estaciones San José (SJS), La Lucha (LCR2), Irazú (ICR) y Urasca (URS); esto asegura una mejor cobertura azimutal de los temblores de la zona. Fue requisito también, que el temblor estuviera registrado en al menos 5 estaciones y se debía contar con 2 o más lecturas de la onda $\mathrm{S}$. Otras restricciones para la selección de los eventos fue que éstos tuvieran una cobertura azimutal mayor a $180^{\circ}$, que el error cuadrático medio (RMS) de las localizaciones fuera menor a $0,5 \mathrm{~s}$ y que los errores de localización fueran inferiores a $2 \mathrm{~km}$ en la componente horizontal y de $3 \mathrm{~km}$ en la vertical. Estos criterios se aplicaron a temblores con formas de onda de registro digital (período 1992-1996).

En lo que respecta a las inversiones del mecanismo focal, se utilizaron temblores con excelente ubicación y cobertura azimutal (Gap < 180) y con un mínimo de 10 polaridades. Para realizarlos se usó la rutina Focmec del Programa SEISAN (Havskov \& Ottemoller, 1999); se buscaron soluciones cada 5 grados, se escogió como inversión de calidad $\mathrm{A}$, a aquella con un número de soluciones menor a 50, todas ellas con poca variación entre sí (es decir, aunque existan varias soluciones, todas ellas indican el mismo tipo de mecanismo). Un evento con mayor Gap, menos polaridades y más de 50 soluciones se considera de calidad B. En este trabajo solamente se utilizaron inversiones de calidad A, porque las de inferior calidad dificultan las interpretaciones en vez de facilitarlas.

Con relación al fallamiento neotectónico incorporado en este estudio, se cumplieron varias fases en su investigación. En primer lugar se revisaron los trabajos previos sobre el mismo, entre los que destacan Montero et al. (1989 a y b), Montero et al. (1991), Woodward \& Clyde (1993), Montero (1994) y GEOMATRIX (1994). Las fallas incorporadas en estos estudios fueron revisadas utilizando las fotos aéreas, buscando confirmar las sugerencias geomorfológicas de fallamiento neotectónico. Durante este proceso se revisaron dentro de la zona de estudio nuevas sugerencias de posibles fallas neotectónicas, que no hubieran sido mencionadas en los trabajos previos o incluso nuevas trazas o cambios en las trazas con respecto a las fallas previamente reconocidas. Se hizo un reconocimiento de campo para confirmar las geoformas y se visitaron afloramientos que podrían aportar evidencias geológicas de que los lineamientos corresponden con fallas activas. Luego de realizar estos análisis se definieron las trazas de las fallas que se muestran en la figura 1. Como se puede observar, la mayoría de las trazas que se han definido en este trabajo se basan en el análisis de evidencias geomorfológicas, por lo que en varios casos las fallas consideradas solo están bien restringidas parcialmente. Además, la relativamente baja velocidad de deslizamiento que sugiere tener la mayoría de ellas, de acuerdo a su débil prominencia, provoca dificultades para su identificación, considerando que nos ubicamos en un ambiente climático tropical.

\section{DESCRIPCIÓN DE LAS FALLAS PRINCIPALES}

A continuación se realiza una descripción resumida de las principales fallas neotectónicas que se han definido en la zona comprendida entre San José y Cartago (Fig. 1).

\section{Falla Escazú (FES)}

Es una falla de rumbo NW que tiene una extensión de 6,5 km y que parece tener levantamiento en su bloque oeste. Fue originalmente reconocida por Montero et al. (1989a). Morfológicamente se destaca porque tiene un escarpe en el lado oeste de la traza, el cual se muestra bastante disectado. También se observan alineaciones de varios ríos y quebradas. Podría tener continuidad con las fallas Belo Horizonte, Jericó y Aserrí.

\section{Falla Belo Horizonte (FB)}

Esta falla fue originalmente reconocida y denominada por Montero et al. (1989a) y Woodward 
\& Clyde (1993), aunque el trazo mostrado en los anteriores trabajos difiere parcialmente con el aquí propuesto. Es una falla corta con una longitud de $3 \mathrm{~km}$, ubicada al pie de los cerros que se encuentran entre el oeste de Alajuelita y Belo Horizonte, al suroeste de la ciudad de San José. Se caracteriza por presentar un cambio fuerte de pendiente, escarpes poco facetados que miran al este y por la posible presencia de ríos descabezados, desviados o desplazados. Evidencias de campo de actividad reciente no han sido encontradas, aunque sus escarpes pronunciados sugieren que es neotectónica; parece estar sísmicamente activa. Podría tener continuidad con la falla Escazú y con la falla Jericó, formando un patrón en echelon (en escalera) o ser parte de una zona transpresiva.

\section{Falla Aserrí}

Fue descrita originalmente y denominada por Montero et al. (1989a) y Woodward \& Clyde (1993). Tiene rumbo NW y una longitud de $4 \mathrm{~km}$. Se caracteriza por presentar silla de falla, facetamiento triangular, posible abanico aluvial desplazado y levantándose del lado suroeste. Muestra una divisoria desplazada dextralmente y tiene forma rectilínea. Esta falla puede tener continuidad con la falla Belo Horizonte y con la falla Jericó. Las evidencias geomorfológicas sobre su actividad neotectónica son bastante fuertes y permiten catalogarla como una falla activa conocida (Montero et al., 1989b).

\section{Falla Jericó}

La falla Jericó se describe por primera vez en este trabajo. Al noroeste puede tener continuidad con las fallas Aserrí y Belo Horizonte. Tiene un rumbo $\mathrm{NW}$, aunque localmente se observan cambios de rumbo. Estos últimos son más evidentes a partir de donde se divide en dos ramales; sus trazas indican que la falla tiene un buzamiento de alto ángulo al SW. Al noroeste se inicia cerca de Alajuelita, pasa por San Juan de Dios de Desamparados, al este de Aserrí y al oeste de El
Llano, donde se divide en dos trazas. La traza este pasa por Jericó y se une con la otra traza cerca del poblado de Alumbre, mientras que la traza oeste pasa por las quebradas Honda y Manzano. Más al sureste, fuera del área de estudio, se continúa con la falla Frailes. Dentro del área de estudio se extiende por cerca de $13 \mathrm{~km}$.

La falla se caracteriza por mostrar múltiples geoformas sugestivas de neotectonismo, como son sillas de falla, escarpes facetados, valles lineales, valle de falla, desplazamiento dextral de divisoria y contraescarpes, entre otros. Fue observada en San Juan de Dios, desplazando suelos recientes. Las evidencias indican que la falla tiene una componente de desplazamiento de rumbo dextral, aunque una componente de levantamiento del lado oeste se infiere en el sector noroeste.

\section{Falla Río Azul (FRA)}

Originalmente fue identificada por Montero et al. (1989a) y Woodward \& Clyde (1993). Sin embargo, la traza que mostramos en este trabajo difiere parcialmente con la identificada en los anteriores trabajos. Es de rumbo este-noreste y tiene una longitud cercana a $5 \mathrm{~km}$. Se caracteriza por la presencia de un valle profundo lineal al pasar por la quebrada Quebradas. Además, presenta sillas de falla, espolón truncado y facetado triangular.

\section{Falla Cipreses y sistema de pliegues asociados}

Corresponde en su extremo oeste y sur con una serie de superficies geomorfológicas abovedadas de rumbo NW a EW, que se ubican entre Granadilla y Tres Ríos. Se considera que estas geoformas se relacionan con una secuencia de pliegues anticlinales y sinclinales, que se pueden asociar con una falla de propagación, como es la falla de Alajuela (Borgia et al., 1990). En esta zona había sido identificado previamente un lineamiento sugestivo de falla reciente denominado Granadilla, de rumbo NW (Montero et al., 1989a; Woodward \& Clyde, 1993). Sin embargo, 
nuestra interpretación es que este corresponde con la traza de un pliegue sinclinal que pasa por donde se ubica el río Pío.

En relación con la edad del fallamiento y plegamiento de esta zona, debe mencionarse que fallamiento con separación normal fue observado desplazando capas de suelo en un afloramiento localizado al norte de San Juan, sobre el sector donde se presenta un escarpe de rumbo E-W. Esto último señala que la falla es activa.

\section{Falla Agua Caliente (FAC)}

La falla Agua Caliente ha sido sugerida como una falla neotectónica desde el trabajo de Dóndoli \& Torres (1954). La ubicación de esta falla ha sido propuesta con diferentes trazas (Dóndoli \& Torres,1954; Montero et al., 1989a; Montero et al., 1991, Montero, 1994). Lo anterior se debe a que la falla tiene una expresión geomorfológica débil en algunos sectores. En algunos de los anteriores trabajos, la falla Agua Caliente se denominó valle de Coris y Guarco. En este trabajo, la ubicamos como una falla de forma sinuosa con rumbo variable entre NW, EW y WNW en sus sectores oeste, central y este respectivamente.

Geomorfológicamente, la falla Agua Caliente se caracteriza por el alineamiento de promontorios truncados, contraescarpes en depósitos recientes, contrastes de vegetación en zonas planas, alineamiento de valles lineales y de fuentes termales y mineralizaciones sulfurosas (valle de Coris y Hervidero), lomos de falla y sillas de falla. En el sector oeste del valle de Coris, la falla fue observada en una trinchera exploratoria cortando depósitos holocénicos (Woodward \& Clyde, 1993). En otra trinchera realizada en una fila de lomos de falla ubicados al sur de Cartago, se encontró depósitos del Cuaternario Superior cortados por fallas inversas asociadas con esta falla (Montero et al., 1991).

La falla Agua Caliente podría iniciarse al oeste con un lineamiento sugestivo de ser falla reciente que tiene rumbo NW y que geomorfológicamente tiene una prominencia débil (denomi- nado lineamiento San Sebastián en Woodward \& Clyde, 1993). El trazo activo de la falla Agua Caliente se inicia con seguridad al sur de la Loma Salitral y se continúa hacia el este con un rumbo oscilante entre EW y WNW. La falla es de movimiento predominante siniestral y tiene una zona transtensiva en el sector entre Quebrada Honda y Bermejo. Asimismo, existe una transpresión local en el sector de Tejar (Montero et al., 1991). En el extremo este, la falla tiene débil prominencia y puede continuar al este hasta terminar contra la falla Navarro (no se muestra en figura 1, ver trazo en Woodward \& Clyde, 1993).

\section{Falla Cangreja}

Esta falla se describe por primera vez en el presente trabajo. Tiene un rumbo NW y una extensión de $6 \mathrm{~km}$. Al noroeste se inicia cerca de Barrancas, al este de Tobosi y al sureste termina al este del poblado de Cangreja. Se encuentra comprendida entre las fallas Agua Caliente y Navarro.

Geomorfológicamente se caracteriza por su trazo rectilíneo, lo cual indica que es de inclinación vertical a subvertical. Además, muestra valles lineales, sillas de falla, trincheras de falla, escarpes facetados y bermas de falla.

\section{Sistema de falla de Ochomogo}

Es un sistema de lineamientos cortos de rumbo NE que se localizan al pie de los cerros de la Carpintera en su sector este. Se evidencian por una prominencia débil a moderada, muestran facetamiento triangular que mira al este y sillas de fallas. Al pasar por Ochomogo parecen haber sido alterados hidrotermalmente los depósitos laháricos cuaternarios que corta.

\section{Falla Orosi}

Es una falla de rumbo NW con una expresión geomórfica prominente en el sector al sur de la falla Navarro, donde muestra un escarpe de falla que mira al este y que posiblemente 
está asociado con una falla de desplazamiento normal. El escarpe tiene un facetamiento triangular con varias generaciones de facetas. Del lado norte de la falla Navarro pierde prominencia. Parece estar desplazada en sentido siniestral por la falla Navarro.

\section{Falla Navarro}

Falla de movimiento siniestral de rumbo ENE que se localiza del lado norte del valle del río Navarro, el cual se puede considerar un valle de falla. Al sur de Paraíso, se evidencia por sus trincheras de falla, sillas de fallas, valles lineales, entre otros. En GEOMATRIX (1994) se presentan evidencias de su desplazamiento reciente, lo cual fue confirmado en trincheras.

\section{SISMICIDAD Y SISMOTECTÓNICA}

\section{Sismicidad histórica}

Como se mencionó previamente, varios terremotos históricos han tenido su epicentro en la zona estudiada y dos de ellos causaron severo daño y muerte. Estos fueron los terremotos que destruyeron a Cartago el 2 de setiembre de 1841 y el 4 de mayo de 1910, con saldo de 16 muertos durante el primer sismo y de 600 en el segundo (Rojas, 1993). Otros temblores importantes fueron el temblor de Alajuelita del 21 de marzo de 1842, el temblor del Tablazo que ocurrió el 13 de abril de 1910, el de San Diego de Tres Ríos del 21 de febrero de 1912 y el de Paraíso del 22 de agosto de 1951. Los epicentros sugeridos para estos temblores se muestran en la figura 1 y se dan más detalles de ellos en el cuadro 1.

Todos estos temblores han tenido su epicentro al sur y sureste de San José, en zonas de fallamiento reconocido; la mayoría de ellos más cerca de Cartago que de San José. Para los terremotos de Cartago y de Tablazo se indica en la figura 1 el área de daños, con las intensidades Mercalli Modificada $\geq$ VII, según Montero \& Miyamura (1981).

\section{Sismicidad reciente}

Las primeras localizaciones de temblores de la zona realizadas por la RSN (período 19761981) aparecen en Montero \& Dewey (1982), Montero \& Morales (1984) y Montero \& Alvarado (1995). Dicha información, que incluye localizaciones de calidad A (círculos rellenos) y B (círculos sin relleno), ha sido incorporada en este trabajo y se presenta en la figura 2. En esta figura se observan tres agrupamientos de sismos, uno en la esquina inferior izquierda (corresponde con los cerros de Escazú), otro entre la falla Jericó y el extremo occidental de la falla Aguacaliente (FAC) y el último al sureste de Higuito. La sismicidad está compuesta en su mayoría por sismos de calidad B con magnitudes entre $-0,1$ y 2,9 grados. De los tres agrupamientos el que mejor se vincula con el fallamiento es el que se ubica al noroeste de Higuito, el cual podría estar asociado

Cuadro 1

Temblores históricos de la zona estudiada (Rojas, 1993)

\begin{tabular}{cllll}
\hline Número & Nombre & Día-mes-año & Mag. (Ms) & Daños \\
\hline 1 & T. Cartago & $02-09-1841$ & $6,0-6,5$ & Destrucción de Carta-go, 38 muertos \\
2 & T. Alajuelita & 1842 & $5,0-5,5$ & Daños en Alajuelita \\
3 & T. Tablazo & $13-04-1910$ & 5,8 & Daños en Desampa-rados \\
4 & T. Cartago & $04-05-1910$ & 6,4 & Destrucción de Cartago y Paraíso, 600 muertos \\
5 & T. Tres Ríos & 1912 & $5,0-5,5$ & Daños en Tres Ríos \\
6 & T. Paraíso & 1951 & 5,4 & Daños en Paraíso y Orosi \\
\hline
\end{tabular}

T: Terremoto, Ms: Magnitud Ms, Ml: Magnitud Local 


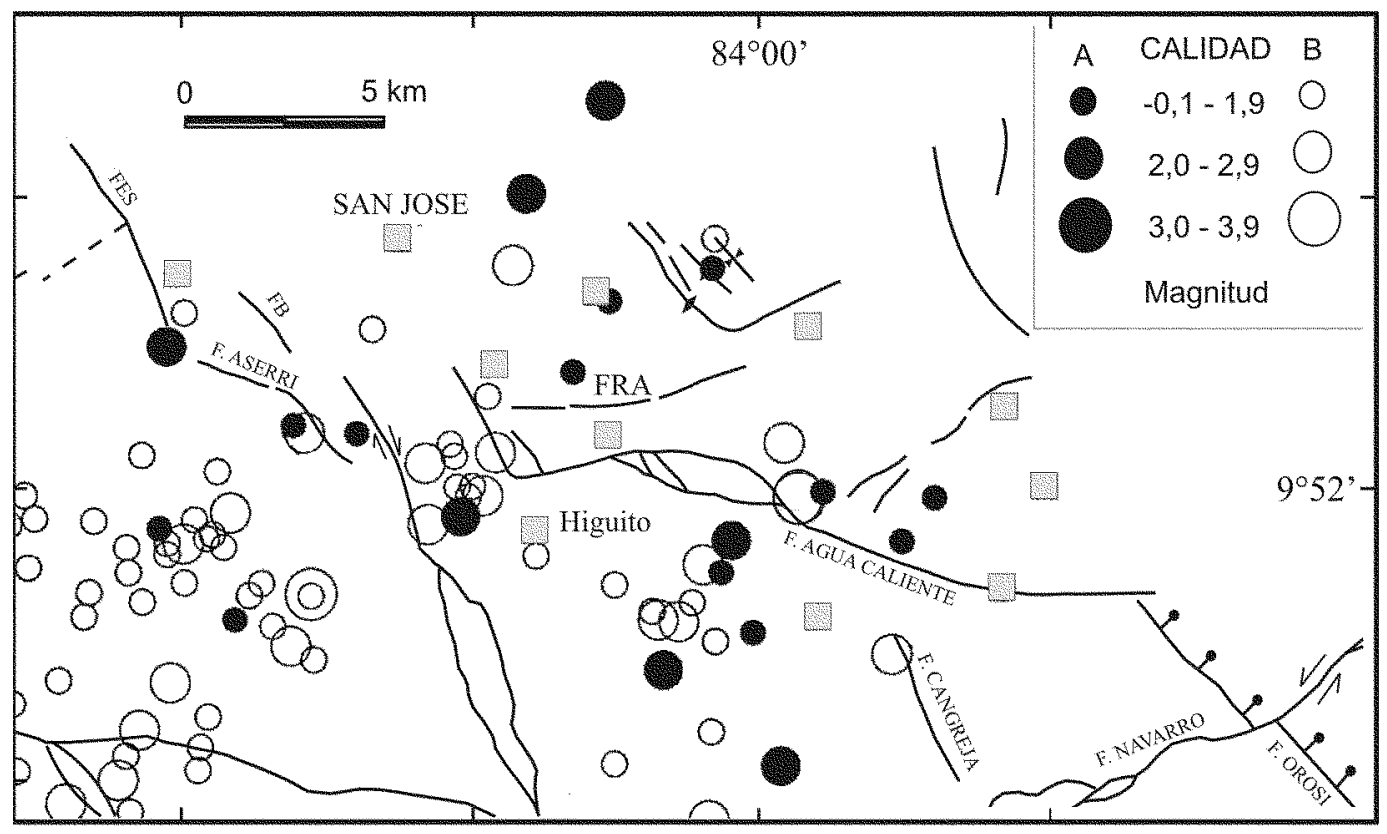

Fig. 2: Sismicidad del período 1976-1981 de acuerdo con Montero \& Dewey (1982), Montero \& Morales (1984) y Montero \& Alvarado (1995).

con la actividad sísmica de las fallas Jericó y Aguacaliente, siendo muy difícil atribuir los temblores a una de las dos en particular. Para los restantes agrupamientos no existen fallas cartografiadas con que asociarlos; en los estudios neotectónicos no se han encontrado evidencias de fallamiento, por lo que podrían relacionarse con fallas poco prominentes. Uno de los eventos de mayor magnitud de esta serie, con localización de calidad B, se ubica en Coris sobre la traza de la falla Aguacaliente. Según estos datos, las fallas de Escazú, Jericó, Orosi y Navarro prácticamente no generaron sismicidad durante el período 1976-1981.

En la base de datos de la Red Sismológica Nacional (RSN: ICE-UCR) hay 470 temblores superficiales que ocurrieron en la zona durante el período 1984 a 1996. La figura 3 muestra la distribución epicentral de estos temblores sin ninguna selección previa. Se incluye esta información para dar una idea de la cantidad de sismos que ha registrado y localizado la RSN en la zona durante el período indicado; la cual se incrementó luego de la intensa actividad sísmica ocurrida en la parte central de Costa Rica entre 1990 y 1993.
Asimismo, el incremento en el número de estaciones sísmicas permitió aumentar el nivel de detección de la RSN durante ese período. Muchos de estos temblores fueron localizados con apenas tres estaciones y por ello no se les puede asignar calidad A. Pese a que esta sismicidad está dispersa por todo el área, su patrón de distribución confirma la existencia de los agrupamientos sísmicos mencionados anteriormente. También permite deducir que en los últimos años ha habido más sismicidad en las fallas de Escazú y Navarro. En cuanto a la magnitud de los temblores, se aprecia que los más abundantes son aquellos de magnitud inferior a 2; hay un número importante de sismos de magnitud 3 y muy pocos de magnitud 4 .

De los 470 temblores de la figura 3, solamente 55 ocurridos en el período 1992-1996, cumplieron los criterios de selección indicados en la metodología y son los que se muestran con círculos de color negro en la figura 4 . Tal como se observa en dicha figura, los temblores seleccionados son en su mayoría de magnitudes bajas, las que varían entre 2 y 3 grados. Solamente tres eventos fueron de magnitud superior a 4,0 grados 


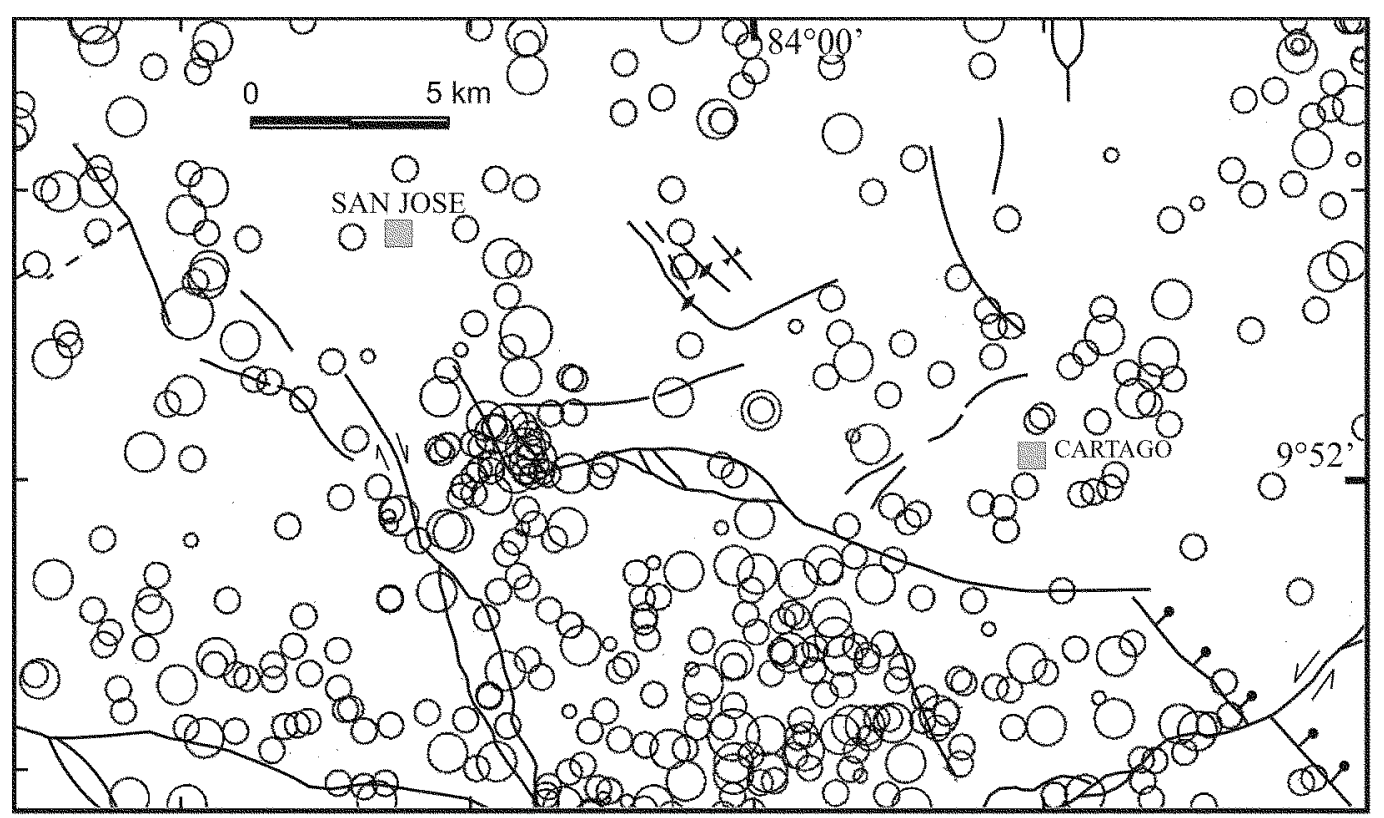

Fig. 3: Sismicidad de profundidad $\leq 30 \mathrm{~km}$ de la zona en el periodo 1984-1996 a partir de la base de datos de la RSN (ICE-UCR). En esta figura el tamaño de los círculos en orden de menor a mayor corresponden con los siguientes rangos de magnitudes: $1-1,9 ; 2-2,9 ; 3-3,9$ y $4-4,9$.

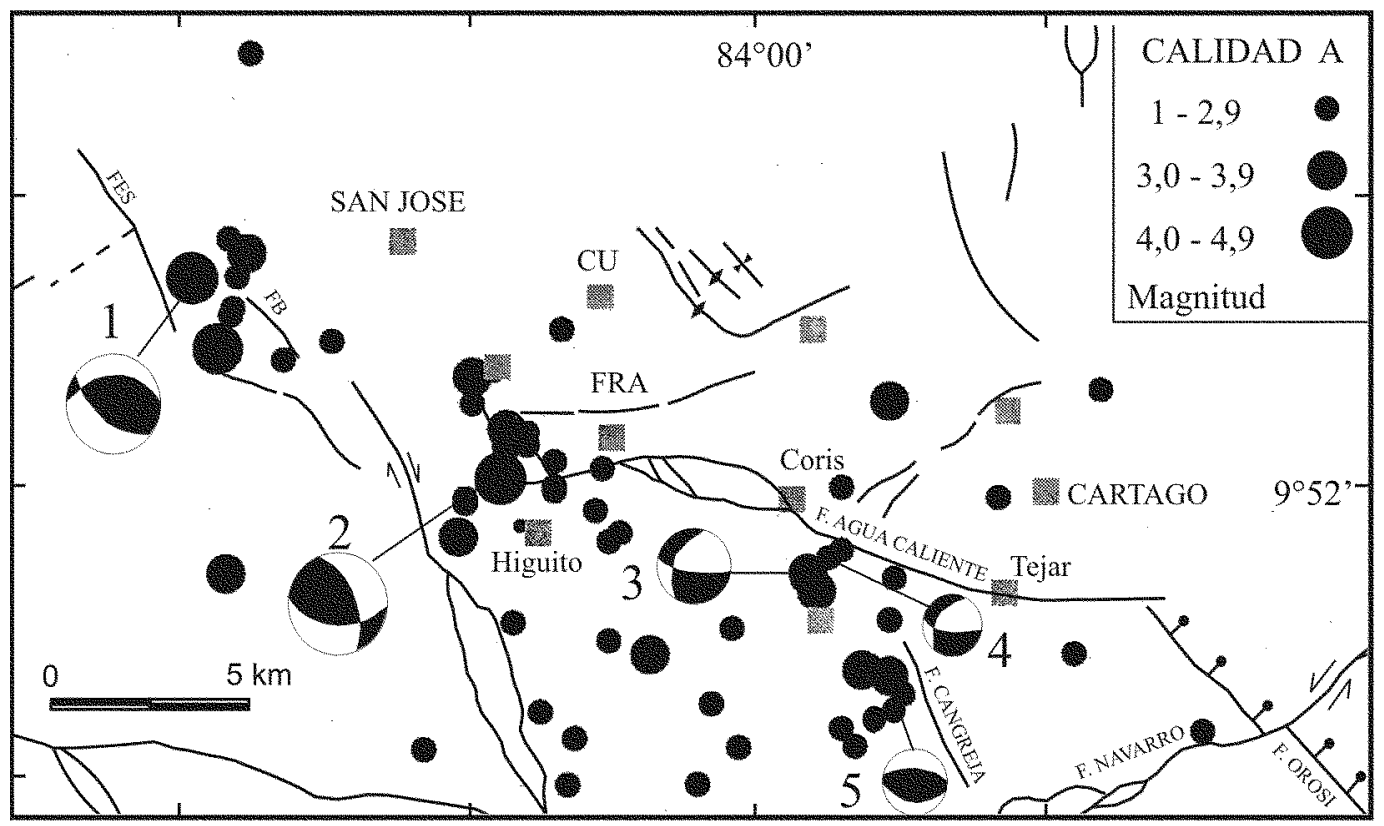

Fig. 4: Sismos seleccionados y relocalizados en este trabajo y mecanismos focales numerados de acuerdo a cuadros 2 y 3 , cuyas polaridades se muestran en la figura 5. Zonas de compresión en negro y de dilatación en blanco y proyección del hemisferio focal inferior. 
(4,0, 4,2 y 4,6), dos de ellos están relacionados con actividad tectónica en las fallas cercanas a Escazú y el otro fue parte de unos enjambres sísmicos que ocurrieron en 1994 en Desamparados. La profundidad de los sismos varía entre 1 y $20 \mathrm{~km}$.

Cinco de los temblores seleccionados reunieron suficiente número de polaridades para hacer inversiones de mecanismo focal (Fig. 5). En el cuadro 2 se dan las localizaciones de los tem-
192/11/03 RSN

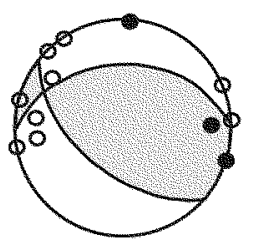

2 96/05/23 RSN-ORO

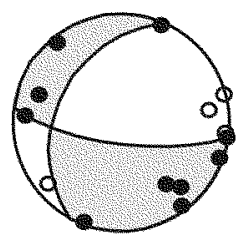

2 94/10/29 RSN-UNA

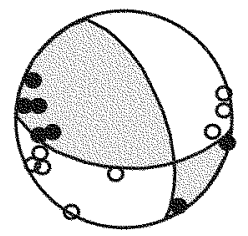

2 96/05/23 RSN-ORO

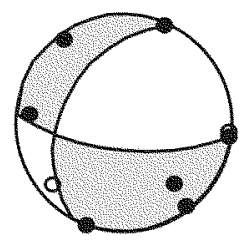

5 96/09/21 RSN-ORO

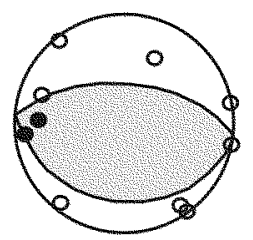

Fig. 5: Soluciones de la inversión de mecanismos focales y distribución de polaridades. Datos de polaridades provienen de RSN (ICE-UCR), OVSICORI de la Universidad Nacional (UNA) y ORO (Red de Orotina de la UCR). blores para los que se hizo inversión del mecanismo focal y en el cuadro 3 se reporta un plano de la solución focal y otros datos de la inversión.

A continuación se realiza una interpretación de la distribución espacial de los sismos con respecto al fallamiento y los mecanismos focales. Consideramos tres agrupamientos principales de temblores (figura 4):

\section{Zona sísmica entre Cartago-Coris y Tobosi}

Un grupo de temblores se localiza al oeste de Cartago, al este de Coris y cerca de Tobosi, donde hay sismos tanto al sur como al norte de esta localidad, con magnitudes que oscilan alrededor de 3,0. Los del sur se ubican al oeste de la falla Cangreja y algunos se ubican lo suficientemente cerca de ésta, como para sugerir que se pueden relacionar con la misma. Sin embargo, también se puede interpretar que se alinean en dirección casi este-oeste, en forma consistente con el rumbo del lineamiento Tobosi (Montero et al.,1989a; no se muestra en la figura 4). Los del norte se ubican cerca de la falla Agua Caliente y del sistema de fallas cortas de Ochomogo.

El mecanismo focal 5 fue realizado para un temblor ubicado al oeste de la falla Cangreja (Fig. 4). Es de tipo inverso con planos nodales de rumbo cercano al E. En esta misma zona, Montero \& Dewey (1982) habían encontrado un mecanismo focal inverso con rumbos similares. Este tipo de fallas no ha sido detectado fotogeológicamente, excepto por el lineamiento Tobosi, que podría ser de este tipo de fallas.

Cuadro 2

Localizaciones de temblores con mecanismo focal de temblores

\begin{tabular}{llllllllllll}
\hline $\mathrm{N}^{\circ}$ & Fecha & Hora & Latitud & Long. & P. $(\mathrm{km})$ & Mag. & Rms & Erln & Erlt & Erlp & Gap \\
\hline 1 & 921103 & $11: 46$ & 9,921 & 84,138 & 06,5 & 4,1 & 0,30 & 0,5 & 0,8 & 0,9 & 88 \\
2 & 941029 & $05: 37$ & 9,867 & 84,064 & 06,6 & 4,2 & 0,30 & 0,5 & 0,8 & 0,5 & 43 \\
3 & 960523 & $06: 31$ & 9,850 & 83,988 & 11,4 & 3,1 & 0,36 & 0,6 & 0,8 & 2,0 & 60 \\
4 & 960523 & $06: 33$ & 9,854 & 83,983 & 10,1 & 2,5 & 0,32 & 0,6 & 0,8 & 1,9 & 59 \\
5 & 960921 & $10: 41$ & 9,815 & 83,971 & 08,6 & 2,9 & 0,27 & 0,6 & 0,8 & 1,6 & 74 \\
\hline
\end{tabular}

P: Profundidad, Mag: Magnitud, Rms: Error cuadrático medio, Erln: Error en la longitud, Erlt: Error en la latitud, Erdp: Error en la profundidad. 
Cuadro 3

Datos de las soluciones focales

\begin{tabular}{lccccccc}
\hline$N^{\circ}$ & Azimut & Buz. & Rake & NP & EP & NS & C \\
\hline 1 & 269 & 40 & 58 & 13 & 1 & 11 & A \\
2 & 253 & 84 & 40 & 16 & 0 & 11 & A \\
3 & 097 & 74 & -53 & 14 & 0 & 17 & A \\
4 & 095 & 70 & -52 & 09 & 0 & 14 & A \\
5 & 095 & 30 & 90 & 11 & 0 & 38 & A \\
\hline
\end{tabular}

Buz: Buzamiento, NP: Número de Polaridades, EP: Error de Polaridades, NS: Número de Soluciones, C: Calidad.

Los mecanismos focales 3 y 4 fueron realizados para un par de eventos que se ubicaron relativamente cerca de la falla Agua Caliente. Si estuvieran relacionados con la misma, indicarían que un desplazamiento siniestral con una componente normal en un plano nodal de rumbo cercano al EW. También existe la posibilidad de que se hayan originado en una falla de rumbo NE, tal como lo sugieren algunos epicentros.

\section{Zona sísmica Patarrá - Higuito}

El segundo agrupamiento importante de temblores se relaciona con tres enjambres sísmicos que ocurrieron al sur de San José, en la zona de San Miguel, Higuito y Patarrá:

\section{Enjambre de junio de 1994}

La actividad se inició el 3 de junio de 1994 cuando vecinos de las Lomas de Salitral empezaron a oír retumbos pero sin sentir temblores. La RSN (ICE-UCR) localizó y reportó como sentidos solamente cuatro eventos de este enjambre, los que Rojas et al. (1994) relacionaron con las fallas Higuito y Salitral (no incluidas en las figuras 1 a 4). En este trabajo se han incorporado las soluciones hipocentrales reportadas por la RSN, encontrándose que el mayor evento (magnitud $3,4)$ se ubica al sur de Desamparados, en un área donde se ubican las fallas Agua Caliente, Río Azul y Jericó (Fig. 4). Los pocos sismos localizados de este enjambre y la cercanía entre las fallas en esta zona, no permiten determinar con certeza cual fue la falla que originó la actividad sísmica.

\section{El enjambre de octubre de 1994}

Entre los días 25 y 31 de octubre de 1994 nuevamente se originó un enjambre de temblores en la misma zona epicentral en donde ocurrió el de junio de ese mismo año. En este caso se generó una secuencia de temblores cuyo máximo evento tuvo magnitud 4,2. En esta ocasión, la energía liberada fue superior a la que se liberó en junio, razón por la cual un mayor número de temblores fue sentido por la población. La RSN reportó 13 temblores sentidos de esta serie (RSN, informe de octubre 1994) y, al igual que en junio, se relacionaron con las fallas Salitral e Higuito. En octubre hubo más temblores de magnitud 3 localizados con soluciones confiables, lo cual permitió definir mejor las fuentes de estos temblores. Sin embargo, dado que los temblores ocurrieron dentro de un área donde se localizan varias fallas, es difícil determinar cual de éstas fue la que generó los sismos. No obstante, la distribución de epicentros cerca de la loma Salitral sugiere que fue la falla Agua Caliente, la que causó la sismicidad de octubre en Desamparados. Sin embargo, no se puede descartar que en las fallas Jericó y Río Azul hayan ocurrido desplazamientos durante ese enjambre, dada la cercanía entre los epicentros y estas fallas.

El mecanismo focal 2 fue realizado para el evento principal de esta secuencia; este corresponde con una solución de falla oblicua con componentes de desplazamiento transcurrente e inverso; el plano nodal al Este coincide con el rumbo de la falla Aguacaliente entre la Loma Salitral y el sur de Cartago, mientras que el plano noroeste concuerda con el rumbo de la falla Agua Caliente donde cambia de rumbo al noroeste, en el sector que en Woodward \& Clyde (1993) se denominó como el Lineamiento San Sebastián. Además, este evento se localiza muy cerca de la falla Jericó que tiene rumbo noroeste. Lo anterior, hace difícil escoger un plano nodal como plano de falla en este mecanismo focal. Sin embargo, considerando el mecanismo focal 2 en 
conjunto con los 3 y 4, parece más apropiado escoger la falla Agua Caliente como la falla donde se generó este enjambre. Así, los planos nodales al Este, son todos de desplazamiento siniestral pero con componente inversa (2) en el sector de la Loma Salitral y normales (3 y 4) en la zona del valle de Coris.

\section{Enjambre de noviembre de 1997}

El 13 de noviembre de 1997 se registró un evento de magnitud 3,7 cerca de la intersección de las fallas Jericó y Aguacaliente; se localizó a $11 \mathrm{~km}$ de profundidad y tuvo intensidad máxima de IV en la zona epicentral. Luego de este evento, se registraron 2 réplicas con magnitud mayor que 3,0 y más de 40 con magnitudes menores a 3,0. Los epicentros de estos temblores se ubican cerca de las fallas Jericó y Agua Caliente, y todo parece indicar que este nuevo enjambre se originó en las mismas. Los eventos de este enjambre no se muestran en la figura 4 .

\section{Zona sísmica de Escazú - Belo Horizonte}

Un tercer grupo de temblores ocurrió cerca de la falla Belo Horizonte, durante noviembre de 1992, enero de 1993 y setiembre de 1993. Estos tienen una tendencia noroeste, en la misma dirección de la falla Belo Horizonte.

El mecanismo focal 1, realizado para un evento ocurrido en esta zona sísmica, corresponde con fallamiento oblicuo inverso-transcurrente y planos nodales de rumbo oeste y noroeste. Obsérvese que si las fallas Escazú y Jericó se relacionan entre sí en la zona donde se ubica Belo Horizonte, se tendría una zona transpresiva, que podría explicar bien la presencia de las fallas Belo Horizonte y Aserrí, con un fallamiento de dirección oeste-noroeste como el sugerido por el mecanismo focal.

\section{Sismicidad dispersa}

En la figura 4 se observa sismicidad dispersa en el sur del área de estudio, entre las fallas
Jericó y Cangreja. También hay epicentros dispersos cerca de Cartago y un evento aislado al sur de la falla Aserrí. En esas áreas no se han identificado aun trazas de fallas neotectónicas en superficie.

\section{Síntesis neotectónica y sismotectónica}

Los rasgos neotectónicos identificados en la zona de estudio, permiten definir tres sistemas principales de fallas. El primero corresponde con el sistema constituido por las fallas Escazú, Belo Horizonte, Aserrí y Jericó, que es de rumbo NW y es de acuerdo a los datos neotectónicos, predominantemente dextral con componentes inversa y normal en diferentes sectores. Un mecanismo focal realizado a lo largo de este sistema de fallas es inverso y posiblemente originado en una zona transpresiva, tal como puede ser la conexión entre las fallas Jericó y la Escazú en la zona entre Alajuelita y Belo Horizonte, donde se ubican las fallas Aserrí y Belo Horizonte.

El segundo sistema corresponde con la falla Agua Caliente, que tiene rumbo variable entre ENE a WNW, pero cuya terminación al oeste parece ser de rumbo NW. Neotectónicamente, se interpreta que la falla es siniestral. Trincheras realizadas cerca de Coris y de Tejar sugieren una fuerte componente de desplazamiento de rumbo con componente normal en Coris e inversa en Tejar (Montero et al., 1991). Tres mecanismos focales realizados en dos sectores diferentes de la falla indican un plano nodal de rumbo cercano al EW y deslizamiento siniestral, aunque con componente inversa cerca de Patarrá y normal cerca de Coris. El lineamiento San Sebastián, si se relaciona con la falla Agua Caliente, podría estar asociado con un cambio de rumbo de esta última falla, y sería transpresivo.

El tercer sistema de fallas esta constituido por la falla Río Azul y el sistema de fallas Cipreses y pliegues asociados. En esta zona, la evidencia neotectónica sugiere que estamos en presencia de un fallamiento inverso con plegamiento asociado, pero también, es de esperar fallamiento transcurrente siniestral a lo largo de fallas de rumbo ENE. 


\section{CONCLUSIONES}

El área estudiada ha sido escenario de 6 temblores históricos con magnitud Ms variable entre 5,2 y 6,4 grados, de foco superficial, ocurridos entre 1841 y 1951, donde el último que causó muertes fue el terremoto de Cartago del 4 de mayo de 1910. El más reciente de estos sismos destructivos fue el terremoto de Paraíso que ocurrió en 1951. En conjunto estos eventos causaron la muerte a alrededor de 700 personas y provocaron mucha destrucción de viviendas.

Durante el período 1976-1996, la actividad sísmica del área estudiada ha sido de relativamente baja magnitud, caracterizada por la ocurrencia de enjambres de temblores de corta duración y con bajo número de eventos. Aunque la sismicidad registrada por la RSN (ICE-UCR) parece estar dispersa por toda el área estudiada, los eventos mejor localizados ocurridos entre 1992 y 1996 (figura 4), se concentran en tres agrupamientos principales, a saber: Tobosi y alrededores, Higuito-Desamparados y Escazú. Los tres agrupamientos de sismos se relacionan con las fallas Cangreja y Aguacaliente y el sistema de fallas Jericó-Aserrí-Belo Horizonte-Escazú. Sin embargo, aparte de los tres focos sísmicos anteriores han ocurrido otros sismos en el Valle Central entre 1976 y 1996, los cuales pudieron relacionarse con las fallas que muestran poca actividad con sismos de calidad A del período 19921996. La dispersión de epicentros observada en mapas elaborados con datos donde no se escogen los sismos con criterios de calidad (Fig. 3), puede deberse a errores de lectura propios de los sistemas analógicos, errores de localización y la inadecuada cobertura azimutal de los epicentros.

Después de analizar la sismicidad y el fallamiento neotectónico del sector del Valle Central comprendido entre San José y Cartago, se concluye que la sismicidad mejor definida se encuentra en la zona de San Miguel-Patarrá, la cual se asocia con la falla Aguacaliente. Esta ha originado tres enjambres sísmicos entre junio de 1994 y noviembre de 1997, precisamente donde la falla podría cambiar de rumbo y en una zona donde se esperaría una alta concentración de esfuerzos tectónicos por la presencia de una aspereza geométrica. Esta falla fue la que posiblemente originó el terremoto de Cartago del 4 de mayo de 1910 (Montero \& Miyamura,1981) y de acuerdo con su longitud, representa la principal amenaza sísmica para la región del Gran Area Metropolitana. Sin embargo, dada la ubicación geográfica y la longitud del sistema de fallas de rumbo NW de Escazú - Belo Horizonte-Aserrí - Jericó, que se ubica al oeste y sur de San José y el sistema de fallas de Cipreses y Río Azul, que se ubica al este de San José, estas también representan una significativa amenaza sísmica, dado que actualmente existe una alta concentración urbana y ocurre una fuerte expansión poblacional en las zonas donde se ubican estas fallas.

\section{AGRADECIMIENTOS}

Este estudio fue financiado por la Vicerrectoría de Investigación, proyecto No. 805-99-259. Se agradece a la Red Sismológica Nacional (RSN, ICE-UCR) por los datos suministrados para este estudio. A Ivonne Arroyo por su colaboración en los estudios de campo y a los correctores anónimos.

\section{REFERENCIAS}

BORGIA, A., BURR, J., MONTERO, W., ALVARADO, G., \& MORALES, L.D., 1990: Fault propagation folds induced by gravitational failure and slumping of the Costa Rica Volcanic Range: Implications for large terrestrial and Martian edifices. - J. Geophy. Res. 95: 14357-14382.

DÓNDOLI, C. \& TORRES, A., 1954: Estudio geoagronómico de la región oriental de la Meseta Central. - 180 págs. Ministerio de Agricultura e Industria. San José.

GEOMATRIX CONSULTANTS, 1994: Informe final Acueducto de Orosi. Sub-estudio de la vulnerabilidad sísmica de la conducción: El Llano a Tres Ríos, Provincia de Cartago, Costa Rica. - 75 págs. [Informe técnico].

HAVSKOV, J. \& OTTEMOLLER, L., 1999: Seisan: The earthquake analysis software. For Windows, Sunos, Solaris and Linux. Version 7.0. - 227 págs. Institute of Solid Earth Physics, Univ. Bergen, Norway.

MONTERO, W., 1983: Aspectos sismológicos y tectónicos del Valle Central de Costa Rica. - En: El Sistema 
Fluvial de Tárcoles, Costa Rica. Estudios preliminares sobre geomorfometría y geofactores básicos. Serie Estudios Geográficos. IGN-CONICIT: 75-90.

MONTERO, W., 1994: Sismicidad y neotectónica. - En: DENYER, P. \& KUSSMAUL, S. (eds.): Atlas Geológico Gran Area Metropolitana. - Ed. Tecnológica de Costa Rica: 147-160.

MONTERO, W., \& ALVARADO, G., 1995: El temblor de Patillos del 30 de diciembre de $1952(\mathrm{Ms}=5,9)$ y el contexto neotectónico de la región del volcán Irazú, Costa Rica. - Rev. Geol. América Central, 18: 25-42.

MONTERO, W., \& DEWEY, J., 1982: Shallow-focus seismicity, composite focal mechanism, and tectonic of the Valle Central de Costa Rica. - Seis. Soc. Amer. Bull. 72: 1611-1626.

MONTERO, W. \& MIYAMURA, S., 1981, Distribución de intensidades y estimación de los parámetros focales de los terremotos de Cartago de 1910, Costa Rica, América Central. - Inf. Sem. IGN, 1981(2): 9-34.

MONTERO, W. \& MORALES, L. D., 1984: Sismotectónica y niveles de actividad microtemblores en el suroeste del Valle Central, Costa Rica. - Rev. Geof. 21: 21-41.

MONTERO, W. \& MORALES, L. D., 1988: Zonificación sísmica del Valle Central. Memorias del $4^{\circ}$ Seminario de Ingeniería Estructural, Colegio Federado de Ingenieros y Arquitectos, San José: 335-351.
MONTERO, W., MADRIGAL, R., MORA, R., SEELEY, M., ALT, J., \& CLINE, M., 1989a: Lineaments suggestive of recent fault activity, escala 1: 50000 [Mapa inédito].

MONTERo, W., MONTAlto, F., Alt, J. \& Cline, M., 1989b: Preliminary hazard map for surface-fault rupture and ground failure, escala 1:50000 [Mapa inédito].

MONTERO, W., OBANDO, L., MORA, R., SALAZAR, L. G. \& LEANDRO, G., 1991: Informe final Proyectos Habitacionales Los Diques y Duarco-Suerre. - 72 págs. Univ. de Costa Rica [Informe interno].

MORALES, L., 1987: Regionalización de la sismicidad de Costa Rica y la crisis sísmica del año 1983. - Geoistmo, I (1): 33-49.

ROJAS, W., 1993: Catálogo de sismicidad histórica y reciente en América Central: Desarrollo y Análisis. - 91 págs. Univ. de Costa Rica, San José [Tésis Lic.].

ROJAS, W., PERALDO, G., \& BARQUERO, R., 1994: El enjambre sísmico de Lomas de Salitral, Cantón de Desamparados, junio de 1994. - 5 págs. Red Sismológica Nacional (ICE-UCR) [Informe interno].

WOODWARD \& CLYDE, 1993: A preliminary evaluation of earthquake and volcanic hazards significant to the major population centers of the Valle Central, Costa Rica. - 89 págs. Woodward-Clyde Federal Services, Maryland [Informe interno]. 